\title{
Multiplicity dependence of (multi)strange baryons in the canonical ensemble with phase shift corrections
}

\author{
Jean Cleymans, ${ }^{1}$ Pok Man Lo $\odot,{ }^{2}$ Krzysztof Redlich, ${ }^{2,3}$ and Natasha Sharma $\odot^{4}$ \\ ${ }^{1}$ UCT-CERN Research Centre and Department of Physics, University of Cape Town, Rondebosch 7701, South Africa \\ ${ }^{2}$ Institute of Theoretical Physics, University of Wroclaw, Wroclaw PL-50204, Poland \\ ${ }^{3}$ Theoretical Physics Department, CERN, CH-1211 Genève 23, Switzerland \\ ${ }^{4}$ Department of Physics, Panjab University, Chandigarh 160014, India
}

(Received 17 September 2020; accepted 10 December 2020; published 20 January 2021)

\begin{abstract}
The increase in strangeness production with charged particle multiplicity, as seen by the ALICE collaboration at $\mathrm{CERN}$ in $p-p, p-\mathrm{Pb}$, and $\mathrm{Pb}-\mathrm{Pb}$ collisions, is investigated in the hadron resonance gas model taking into account interactions among hadrons using $S$-matrix corrections based on known phase shift analyses. Strangeness conservation is taken into account in the framework of the canonical strangeness ensemble. A very good description is obtained for the variation of the strangeness content in the final state as a function of the number of charged hadrons in the midrapidity region using the same fixed temperature value as obtained in the most central $\mathrm{Pb}-\mathrm{Pb}$ collisions and with a fixed strangeness suppression factor $\gamma_{s}=1$. It is shown that the number of charged hadrons is linearly proportional to the volume of the system. For small multiplicities the canonical ensemble with local strangeness conservation restricted to midrapidity leads to a stronger suppression of (multi)strange baryons than seen in the data. This is compensated by introducing a global conservation of strangeness in the whole phase-space which is parametrized by the canonical correlation volume larger than the fireball volume at the midrapidity. The results on comparing the hadron resonance gas model with and without S-matrix corrections, are presented in detail. It is shown that the interactions introduced by the phase shift analysis via the $S$-matrix formalism are essential for a better description of the yields data.
\end{abstract}

DOI: 10.1103/PhysRevC.103.014904

\section{INTRODUCTION}

The analysis of data on hadron yields produced in heavy-ion collisions covering a broad range of energies in fixed-target and collider experiments, confirms that produced hadrons originate from a thermal fireball formed in such collisions [1-8]. The yields of produced hadrons are quantified by the statistical operator of the hadron resonance gas model (HRG) with a common freezeout temperature $T_{f}$ and chemical potentials $\vec{\mu}_{f}$ associated with the conserved charges. The volume of the fireball is fixed such as to reproduce the multiplicities of hadrons at a given collision energy $\sqrt{s}$. The thermal freezeout parameters of the produced fireball were shown to be uniquely linked to the collision energy [1,9].

The description of particle production in nucleus-nucleus collisions in the framework of the HRG is particularly transparent at CERN Large Hadron Collider (LHC) energies. There, at midrapidity, particles and antiparticles are produced in pairs, thus all chemical potentials vanish. Consequently,

Published by the American Physical Society under the terms of the Creative Commons Attribution 4.0 International license. Further distribution of this work must maintain attribution to the author $(s)$ and the published article's title, journal citation, and DOI. Funded by $S C O A P^{3}$. the chemical freezeout of all hadrons is quantified by the temperature and the volume of the fireball only.

An impressive overall agreement has been obtained between the measured particle yields by the ALICE collaboration for the most central $\mathrm{Pb}-\mathrm{Pb}$ collisions and the HRG model results [1,2]. The agreement spans nine orders of magnitude in abundance values, encompassing all measured mesons and baryons, as well as light nuclei and hypernuclei and their antiparticles. The analysis was further successfully extended to heavy flavor production by accounting for the initially produced charm quark pairs and their conservation laws $[1,10,11]$.

For the most central $\mathrm{Pb}-\mathrm{Pb}$ collisions, the best description of the ALICE data on yields of particles in one unit of rapidity at midrapidity was obtained at $T_{f}=156.6 \pm 1.7 \mathrm{MeV}[1,5]$. Remarkably, this value of $T_{f}$ coincides within errors with the pseudocritical temperature $T_{c}=156.5 \pm 1.5 \mathrm{MeV}$ obtained from first principles lattice QCD (LQCD) calculations [12], albeit with the possibility of a broad transition region [13]. Furthermore, assuming that the net-charge probability distributions follow the Skelam [14] or generalized Skelam distribution function [15], the ALICE data on different particle yields have been directly compared with LQCD results on charge fluctuations and correlations [16]. The results of this comparison have shown that the susceptibilities are consistent within errors with the results obtained from LQCD at the chiral crossover point. This provides strong evidence for the 
observation that in central $\mathrm{Pb}-\mathrm{Pb}$ collisions all hadrons and their bound states are originating from the hadronized QGP. This conclusion can also be extended to lower collision energies, since freezeout conditions in central heavy-ion collisions were shown to closely follow the chiral crossover at finite baryon chemical potential as calculated in LQCD. A comparison between LQCD and HRG has also been presented in [17] where a higher estimate for the pseudocritical temperature was obtained.

One of the consequences of confinement in QCD is that at lower temperatures $T \leqslant T_{c}$, physical observables require a representation in terms of hadronic states. On the other hand, the successful description of hadron yields in heavy-ion collisions by the HRG and the coincidence of the freezeout and chiral crossover temperatures suggests that the statistical operator of HRG is a good approximation to QCD thermodynamics in the confined phase. Indeed, a direct comparison of the equation of state (EOS) of LQCD and the HRG model has shown that they closely coincide in the hadronic phase, both at vanishing and at small finite $\mu_{B}[18,19]$. These results provide strong support for the view that matter produced in central heavy-ion collisions is a QCD medium in thermal equilibrium described by the HRG statistical operator in the hadronic phase. There are, however, some limitations to the HRG description of QCD thermodynamics which are identified in the context of LQCD as well as in the description of hadron production yields data at the LHC in heavy-ion collisions [5,20,21].

Recent results of LQCD on second-order fluctuations and correlations $\chi_{P Q}$ of conserved charges $P$ and $Q$ allow to identify the HRG approximation in different sectors of hadronic quantum numbers. In particular, in this context it was shown, that at $T \simeq T_{c}$ the HRG underestimates the baryon-strange $\chi_{B S}$ and overestimates the baryon-charge $\chi_{B Q}$ correlations $[21,22]$. In the HRG, the main contribution to $\chi_{B Q}$ is due to protons and resonances which decay into protons, thus too large a value of $\chi_{B Q}$ indicates an excess of protons at $T \simeq T_{C}$. Indeed the HRG analysis of ALICE data in central heavy-ion collisions at collision energy $\sqrt{s}=2.72 \mathrm{TeV}$ has shown that the HRG predicts about $25 \%$ more protons and antiprotons than measured by the ALICE collaboration in central $\mathrm{Pb}-\mathrm{Pb}$ collisions at the LHC $[1,5]$. This constitutes the much-debated "proton-yield anomaly" in the heavy-ion collisions at the LHC.

The partition function of the HRG is evaluated in the grand canonical (GC) ensemble as a mixture of ideal gases of all stable hadrons and resonances as reported by the Particle Data Group (PDG) [23]. A very comprehensive analysis of the influence of hadronic resonances expected from lattice QCD but not listed in the PDG has been presented in [20,24].

In the spirit of the $S$-matrix formalism [21,25-29], which provides a theoretical framework to implement interactions in a dilute many-body system in equilibrium, the presence of resonances corresponds to attractive interactions among hadrons. However, many of the simplifying assumptions of attractive interactions implicit in the HRG model are not necessarily consistent with hadron scattering data. In particular, for an accurate determination of interaction effects, a proper resonance invariant mass distribution and the presence of many
TABLE I. The list of interaction channels included in the coupled-channel PWA describing the $|S|=1$ hyperon system by the Joint Physics Analysis Center (JPAC) Collaboration [70]. Note that $\bar{K}^{*}$ is spin one and together with a nucleon can couple to spin $1 / 2$ $\left(\right.$ denoted $\left.\bar{K}_{1}^{*} N\right)$ and spin $3 / 2\left(\right.$ denoted $\left.\bar{K}_{3}^{*} N\right)$. Subindices \pm represent the higher and lower orbital angular momentum states which couple to a given partial wave.

\begin{tabular}{lccccc}
\hline \hline channel & elastic & channel & quasielastic & channel & unitarity \\
\hline 1 & $\bar{K} N$ & 6 & $\bar{K}_{1}^{*} N$ & 15 & $\pi \pi \Lambda$ \\
2 & $\pi \Sigma$ & 7 & {$\left[\bar{K}_{3}^{*} N\right]_{-}$} & 16 & $\pi \pi \Sigma$ \\
3 & $\pi \Lambda$ & 8 & {$\left[\bar{K}_{3}^{*} N\right]_{+}$} & & \\
4 & $\eta \Lambda$ & 9 & {$[\pi \Sigma(1385)]_{-}$} & & \\
5 & $\eta \Sigma$ & 10 & {$[\pi \Sigma(1385)]_{+}$} & & \\
& & 11 & {$[\bar{K} \Delta(1232)]_{-}$} & & \\
& & 12 & {$[\bar{K} \Delta(1232)]_{+}$} & & \\
& 13 & {$[\pi \Lambda(1520)]_{-}$} & & \\
& & 14 & {$[\pi \Lambda(1520)]_{+}$} & & \\
\hline \hline
\end{tabular}

nonresonant contributions have to be included to be consistent with scattering data. This can be done systematically within the $S$-matrix approach where two-body interactions are, via the empirical scattering phase shifts, included to construct the leading interaction term in the virial expansion of the grand canonical potential [25-32]. The resulting interacting density of states is then folded into an integral over thermodynamic distribution functions, which, in turn, yields the contributions from interactions to a particular thermodynamic quantity.

The $S$-matrix approach has been applied to study the baryon-charge susceptibility $\chi_{B Q}$ in a thermal medium [21]. It was demonstrated that the implementation of the empirical pion-nucleon phase shifts is crucial for the proper interpretation of the LQCD result. Also in the analysis of observables involving nucleons in ultrarelativistic nucleus-nucleus collisions, a careful treatment of pion-nucleon interactions in the partition function of the HRG model could resolve the proton-yield anomaly [5]. Furthermore, in the strange baryon sector of HRG, the improvement of interactions within the $S$-matrix formalism was shown to increase the strange-baryon correlations $\chi_{B S}$ towards the LQCD value at $T \sim T_{c}$ [22]. The coupled-channel analysis naturally incorporates some additional hyperon states beyond those listed in the PDG [23], thus supporting the importance of these states in explaining the LQCD results [20,33].

It is clear that the $S$-matrix scheme can improve the HRG model in approximating the QCD partition function in the hadronic phase, thus producing a more accurate description of the measured particle yields in heavy-ion collisions.

A qualitative display of the $S$-matrix corrections (relative to the HRG baseline) is shown in Fig. 1. The trend of the overall correction is clear: a reduction in the proton yield and an enhancement in the $\Lambda+\Sigma^{0}$ yields. The corrections can reach $\approx-25 \%(\approx+23 \%)$ for protons $\left(\Lambda+\Sigma^{0}\right.$ baryons $)$ at the LHC freezeout conditions. The shaded region (in gray) shows the $S$-matrix scheme implemented with different levels of improvement, e.g., from including only elastic and quasielastic scatterings (black solid line), to the full list of channels (black 

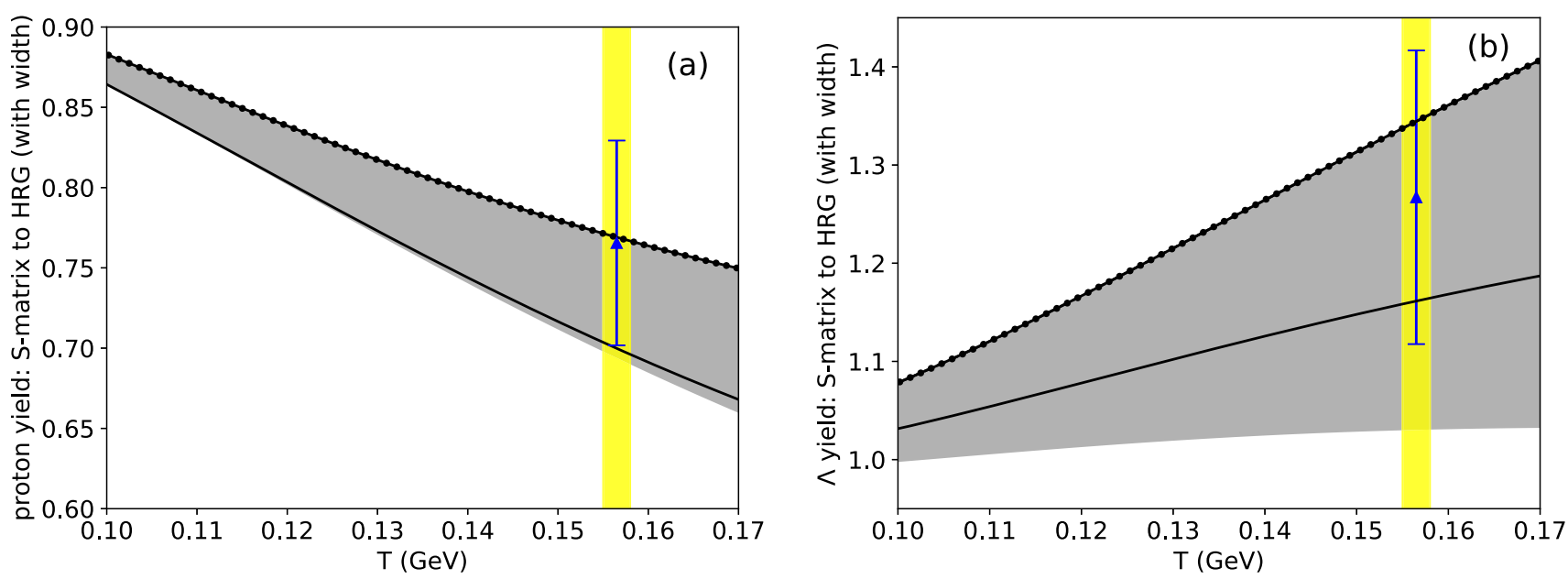

FIG. 1. The ratios of proton (left) and $\Lambda+\Sigma^{0}$ (right) yields in the $S$ matrix and the HRG baseline results. The yellow band signifies the range of pseudocritical temperatures $T_{c}=156.5 \pm 1.5 \mathrm{MeV}$ [12]. The shaded region (in gray) shows the $S$-matrix scheme implemented with different levels of approximation, e.g., from including only elastic and quasielastic scatterings (black solid line), to the full list of channels (black points). See Table I and the discussion in Sec. III. These results can be interpreted as the correction factors for a statistical model provided that the same HRG baseline is employed. (Here, it is THERMUS in its default setting [34].) In all figures we follow the convention to use $\Lambda$ to signify $\Lambda+\Sigma^{0}$ unless otherwise specified.

points). A detailed composition analysis of the hadron yields under the $S$-matrix scheme will be presented in Sec. III.

In the usual formulation of the HRG model, the conservation laws are implemented in the GC ensemble where they are fulfilled on average and are controlled via chemical potentials linked to the conserved quantum numbers. In heavy-ion collisions, however, the conservation of quantum numbers is fulfilled exactly as they are fixed by the initial conditions. Focusing on strangeness production at the LHC, the total strangeness $S$ is exactly zero in the full phase space. Consequently, the constraint of $S=0$ can, in general, influence strangeness production in the acceptance region, which is usually a slice of one unit of rapidity, at midrapidity. The exact and global conservation of net-baryon numbers was also recently shown to be crucial when discussing fluctuations of conserved charges in heavy-ion collisions in a given acceptance region [35-37].

To account for exact charge conservation the HRG thermodynamic potential has to be formulated in the canonical (C) ensemble [38-45] which implies suppression of charged particle yields relative to their GC values. The HRG model formulated in $\mathrm{C}$ ensemble has provided a very useful framework for the centrality and system-size dependence of particle production in this particularly strangeness production and suppression $[41,42,46]$. The applicability of the model in small systems like $p-p$ collisions [47], $p-A$ [48], and $e^{+} e^{-}$annihilation has also been successfully discussed in the literature [49,50].

Recently the ALICE collaboration has observed an interesting systematic behavior in particle production yields at LHC energies, pointing out that the ratios of identified particles to pions depend solely on the charged-particle multiplicity $d N_{\mathrm{ch}} / d \eta$, regardless of system type and collision energy $[8,51-56]$. It is particularly interesting in these data that the evolution of strange particle yields with $d N_{\mathrm{ch}} / d \eta$ shows patterns that are common in $p-p, p-\mathrm{Pb}$, and $\mathrm{Pb}-\mathrm{Pb}$ collisions. The integrated yields of strange and multistrange particles, relative to pions increases with the charged-particle multiplicity and the enhancement becomes more pronounced with increasing strangeness content. Such a pattern of enhancement of (multi)strange hadrons with the number of charged particles is qualitatively similar to what has been observed previously by the WA97 and NA57 collaboration at SPS energies at CERN [57,58] and by the STAR collaboration at the BNL Relativistic Heavy Ion Collider (RHIC) $[59,60]$.

The strangeness enhancement in high-multiplicity $p-p$ collisions observed by the ALICE collaboration and the relation of these data to $p-A$ and $A-A$ collisions cannot be explained quantitatively by general purpose QCD Monte Carlo models $[52,61]$. On the other hand, the first analysis of data within the thermal model with an exact strangeness conservation has shown that the ALICE data are following the model expectations $[48,53,62-64]$. Actually, the observed properties of multi(strange) hadrons with charged-particle multiplicity was predicted in the context of the thermal model as being due to the canonical suppression effect $[42,46]$. Indeed, at the LHC, the canonical suppression depends on the total density of strange particles $n_{S}(T)$ and the correlation volume parameter $V_{C}$ which quantifies the range of strangeness conservation and can be parametrized by $d N_{\mathrm{ch}} / d \eta$. For large $V_{C}$, the canonical suppression factor tends to unity, and particle yields are approaching their $\mathrm{GC}$ values. This is, e.g., the case for central heavy-ion collisions at the LHC, where the GC HRG provides an excellent description of the yields. However, in events with small charged particle multiplicities where $V_{C}$ is small, the canonical corrections cannot be neglected. The characteristic prediction of the HRG model in the C ensemble was an increasing suppression of strange particle yields with decreasing collision energy and collision centrality, as well as with increasing strangeness content of hadrons $[42,46]$.

The main objective of this paper is to apply the $S$-matrix extended HRG model to analyze data obtained by the ALICE 
collaboration on charged-particle multiplicity in $p$ - $p$ collisions at $7 \mathrm{TeV}$ [52] as well as $13 \mathrm{TeV}$ [61], in $p$ - $\mathrm{Pb}$ collisions at $5.02 \mathrm{TeV}[8,53]$ and in $\mathrm{Pb}-\mathrm{Pb}$ collisions at $2.76 \mathrm{TeV}$ [54-56], all in the central region of rapidity.

We focus on yields of (multi)strange hadrons and discuss strangeness suppression as a function of $d N_{\mathrm{ch}} / d \eta$ and strangeness content of hadrons. To this end, we formulate the HRG model in the canonical ensemble of strangeness conservation and account for differences between the fireball volume at midrapidity $V_{A}$ and the correlation volume $V_{C}$ required for exact global strangeness conservation $[39,42,65,66]$. We include the $S$-matrix corrections to the hyperon yields, employing an existing coupled-channel study involving $\pi \Lambda$, and $\pi \Sigma$ interactions in the $S=-1$ sector. We include the $S$-matrix corrections to proton production by using the empirical phase shifts of $\pi N$ scattering and the contribution of hyperons to proton yield.

We will show that the yields of (multi)strange baryons versus $d N_{\mathrm{ch}} / d \eta$ measured by the ALICE collaboration follow the expectations of thermal production with the canonical suppression due to exact strangeness conservation at fixed temperature $T \simeq 156.5 \mathrm{MeV}$, that is consistent with the chiral crossover in LQCD and with a fixed strangeness suppression factor $\gamma_{s}=1$. The yields of (multi)strange hadrons are quantified for different $d N_{\mathrm{ch}} / d \eta$ within one standard deviation. Furthermore, increasing suppression with increasing strangeness content of baryons as a function of $d N_{\mathrm{ch}} / d \eta$ follows patterns obtained recently by the ALICE collaboration. We will discuss the $S$-matrix extended HRG model results in the $\mathrm{C}$ ensemble on yields of (multi)strange baryons, protons, and kaons normalized to pion multiplicity for different $d N_{\mathrm{ch}} / d \eta$, as well as, normalized to $\Lambda$ yield to indicate the importance of $S$-matrix corrections. Our results show, that the thermal origin of particle yields observed in the most central heavy-ion collisions at the LHC [1] can be extended to events with decreasing $d N_{\mathrm{ch}} / d \eta$. Furthermore, the observed scaling of hadron yields with $d N_{\mathrm{ch}} / d \eta$ for different colliding systems is a natural consequence of the HRG model with exact conservation of strangeness. These results provide further evidence for the thermal origin of particle production at the LHC in $p-p$, $p-A$, and $A-A$ collisions at a common $T_{f} \simeq T_{c}$.

The paper is organized as follows. In Sec. II, we introduce the HRG model in the canonical ensemble. In Sec. III, we discuss the $S$-matrix corrections to proton and $\Lambda$ yields. In Sec. IV, we introduce the model comparison with LHC data. In Sec. V, we present our summary and conclusions.

\section{STRANGENESS PRODUCTION WITH CANONICAL SUPPRESSION}

In the GC ensemble, the quantum numbers are conserved on average and are implemented using the corresponding chemical potentials $\vec{\mu}$ linked to conserved charges $\vec{Q}$. The partition function depends on thermodynamic quantities and the Hamiltonian describing the system

$$
Z_{\mathrm{GC}}=\operatorname{Tr}\left[e^{-(H-\vec{\mu} \cdot \vec{Q}) / T}\right]
$$

In the framework of the HRG model considered here and in the Boltzmann approximation, the above leads to

$$
\ln Z_{\mathrm{GC}}(T, \vec{\mu}, V)=\sum_{i} g_{i} V \int \frac{d^{3} p}{(2 \pi)^{3}} \exp \left(-\frac{E_{i}-\vec{q}_{i} \vec{\mu}}{T}\right)
$$

where $g_{i}$ is the spin degeneracy factor of particle $i$ and $E_{i}$ its energy, $V$ is the fireball volume, $\vec{\mu}_{i}$ are chemical potentials associated with conserved charges $\vec{q}_{i}$ carried by particle $i$. The sum is taken over all stable hadrons and resonances as well as their antiparticles. The thermal yield of particle $i$ in the fireball is given by

$$
\left\langle N_{i}\right\rangle_{T}=V g_{i} \int \frac{d^{3} p}{(2 \pi)^{3}} \exp \left(-\frac{\sqrt{p^{2}+m_{i}^{2}}}{T}\right),
$$

where all chemical potentials were set to zero, as relevant for the beam energies at the LHC considered here. We also introduce the density of particle $i$, as $n_{i}=\left\langle N_{i}\right\rangle_{T} / V$.

To get the total multiplicity of a hadron $i$ one has to add resonances decaying to particle species $i$,

$$
\left\langle N_{i}\right\rangle=\left\langle N_{i}\right\rangle_{T}+\sum_{j} \operatorname{Br}(j \rightarrow i)\left\langle N_{j}\right\rangle_{T},
$$

where $\operatorname{Br}(j \rightarrow i)$ is the decay branching ratio of resonance $j$ to particle $i$. To include the width $\gamma_{j}$ of a resonance $j$ with mass $m_{j}$ in Eq. (4), its thermal yield is calculated from

$$
\left\langle N_{j}\right\rangle_{T}=V g_{j} \int \frac{d^{3} p}{(2 \pi)^{3}} \int_{m_{\mathrm{th}}}^{\infty} \frac{d M}{2 \pi} e^{-\sqrt{p^{2}+M^{2}} / T} \Gamma_{j}(M),
$$

where $\Gamma_{j}(M)$ is the relativistic Breit-Wigner distribution function

$$
\Gamma_{j}(M)=\frac{4 M^{2} \gamma_{j}}{\left(M^{2}-m_{j}^{2}\right)^{2}+M^{2} \gamma_{j}^{2}}
$$

and $m_{\mathrm{th}}$ is the value of the invariant mass at threshold for a give decay channel of the resonance.

The results, summarized in Eqs. (3)-(5), extended to quantum statistics, constitute the HRG model in the GC ensemble with all chemical potentials $\vec{\mu}=0$, i.e., the system is charge neutral. Such a model has been applied to quantify thermalization and particle production in most central heavy-ion collisions at the LHC. However, it is already well established, that the GC model can overestimate the yield of particles. This is particularly the case if data are taken in low multiplicity events where the fireball volume is small or at low collision energies where the temperature is low. In such cases, a thermal description requires exact implementation of charge conservation which is usually described in the C ensemble [38-44].

In the following, we focus on exact strangeness conservation and assume that all other quantum numbers are conserved on average in the GC ensemble with $\vec{\mu}=0$. As mentioned earlier, this is a good approximation for the thermal description of the collision fireball produced at LHC energies. In this case, the canonical ensemble with exact implementation of strangeness conservation and total strangeness $S=0$ is 
achieved by introducing a delta function under the trace in Eq. (1),

$$
Z_{S=0}^{C}=\operatorname{Tr}\left[e^{-H / T} \delta_{(S, 0)}\right] .
$$

For a noninteracting Hamiltonian and after performing the Fourier decomposition of the delta function, the canonical partition function can be written in the following integral representation:

$$
Z_{S=0}^{C}=\frac{1}{2 \pi} \int_{-\pi}^{\pi} d \phi \exp \left(\sum_{s=-3}^{3} S_{S} e^{i s \phi}\right),
$$

where $S_{s}=\sum_{k} z_{k, s}$ and the sum is taken over all particles and resonances that carry strangeness $s$. The one-particle partition function is defined as $z_{k, s}=V_{C} n_{k}^{s}(T)$ with the particle density $n_{k}^{s}(T)$ as in Eq. (3). In view of $\vec{\mu}=\overrightarrow{0}$ the thermal phase space of particles is the same as for antiparticles, i.e., $S_{s}=S_{-s}$. The volume $V_{C}$ is the volume where exact strangeness conservation $S=0$ is fulfilled.

The strangeness canonical partition function in Eq. (8) can also be expressed as a series of Bessel functions [40,42,44,67],

$$
Z_{S=0}^{C}=\sum_{n, p=-\infty}^{\infty} I_{n}\left(S_{2}\right) I_{p}\left(S_{3}\right) I_{-2 n-3 p}\left(S_{1}\right) .
$$

The yields of strange particles are usually measured in a given acceptance region, often corresponding to a restricted region in rapidity space. Consequently, the single-particle partition functions $z_{k, s}$ should be split into two parts, $z_{k, s}=z_{k, s}^{A}+$ $z_{k, s}^{R}$, where $z_{k, s}^{A}$ stands for a particle in the acceptance and $z_{k, s}^{R}$ for those outside the acceptance region [35]. Furthermore, we parametrize $z_{k, s}^{A}=V_{A} n_{k}^{s}(T)$, where $V_{A}$ is the volume in the acceptance window.

For the calculation of the mean multiplicity of particle $k$ carrying strangeness $s$ in the acceptance, one introduces the auxiliary parameter $\lambda_{k}^{A}$ in Eq. (8) by replacing $z_{k, s}^{A} \rightarrow \lambda_{k}^{A} z_{k, s}^{A}$. The resulting mean multiplicities in the canonical ensemble in a given experimental acceptance are obtained from

$$
\left\langle N_{k}^{s}\right\rangle_{A}=\left.\lambda_{k}^{A} \frac{\partial \ln Z_{S=0}^{C}}{\partial \lambda_{k}^{A}}\right|_{\lambda_{k}^{A}=1}
$$

in the following form:

$$
\begin{aligned}
\left\langle N_{k}^{s}\right\rangle_{A}= & V_{A} n_{k}^{s}(T) \frac{1}{Z_{S=0}^{C}} \\
& \times \sum_{n, p=-\infty}^{\infty} I_{n}\left(S_{2}\right) I_{p}\left(S_{3}\right) I_{-2 n-3 p-s}\left(S_{1}\right) .
\end{aligned}
$$

It is thus clear, that global and exact strangeness conservation constrained to $S=0$ in the full phase-space influences yields of strange particles in the experimental subspace. The yields are quantified by the temperature and two volume parameters: the volume of the system in the acceptance $V_{A}$ and the correlation volume $V_{C}$ of global strangeness conservation which appear in the arguments of the Bessel functions. Furthermore, to get the total multiplicity of a given strange particle in the $\mathrm{C}$ ensemble, the decays of resonances have to be added as in
Eq. (4), albeit with thermal contribution of strange resonances calculated from Eq. (10).

The first two terms in Eq. (11) constitute the yields of strange particles in the GC ensemble as introduced in Eq. (3), whereas the last terms describe the strangeness canonical corrections. To identify their contribution and dependence on particle strange quantum number $s$, one considers only the leading terms, which correspond to $p=n=0$ in the series in Eqs. (9) and (11),

$$
\left\langle N_{k}^{s}\right\rangle_{A} \simeq V_{A} n_{k}^{s}(T) \frac{I_{s}\left(S_{1}\right)}{I_{0}\left(S_{1}\right)}
$$

Thus, the ratio of $I_{s}\left(S_{1}\right) / I_{0}\left(S_{1}\right)$ is just the suppression factor which decreases in magnitude with increasing $s$ of hadrons and with decreasing thermal phase-space occupied by strange particles, as described by the argument $S_{1}$ of the Bessel functions. A decrease of $S_{1}=V_{C} \sum_{k} n(k, T)$ is due to decreasing $T$, thus also $\sqrt{s}$, or decreasing $V_{C}$ which scales with charged particle density. These are the main properties of strangeness canonical suppression that have been introduced [42] to describe thermal production of multi(strange) hadrons in heavy-ion collisions.

In the following, we apply the HRG model described above in the $\mathrm{C}$ ensemble to quantify production of (multi)strange hadrons and their behavior with charged particle multiplicity as observed by the ALICE collaboration in different colliding systems and collision energies at the LHC. To this end we also correct the HRG model with a more complete implementation of interactions within the $S$-matrix formalism as described in the next section.

\section{III. $S$ MATRIX AND HRG}

Interactions among hadrons modify the density of states (DOS) of a thermal system and hence the thermal abundances of hadron states $[25,26,31,68]$. In the scattering matrix ( $S$-matrix) formulation of statistical mechanics, an effective spectral function $B(M)$ describing the DOS can be computed from the $S$ matrix [25]:

$$
B(M)=\frac{1}{2} \operatorname{Im} \operatorname{Tr}\left[S^{-1} \frac{\partial}{\partial M} S-\left(\frac{\partial}{\partial M} S^{-1}\right) S\right]
$$

where $M$ is the center-of-mass energy of the system. The quantity $B(M)$ summarizes the various interactions among the scattering channels. For the simple case of a single-channel, two-body scattering, the phase shift $\delta(M)$ uniquely identifies the DOS due to the presence of an interaction. For example, when the interaction is dominated by a single resonance of mass $m_{\text {res }}$ and width $\gamma(M)$, the resonant phase shift can be written as

$$
\delta_{\text {res }}(M)=\tan ^{-1} \frac{M \gamma(M)}{m_{\mathrm{res}}^{2}-M^{2}}
$$

The effective spectral function $B$ assumes the standard BreitWigner form upon neglecting the energy dependence of the 
numerator $(M \gamma(M)) \rightarrow\left(M \gamma_{\text {bw }}\right)$ in Eq. (14), such that

$$
\begin{aligned}
B_{\mathrm{res}}(M) & =2 \frac{d}{d M} \delta_{\mathrm{res}}(M) \\
& \approx 2 M \times \frac{2 M \gamma_{\mathrm{bw}}}{\left(M^{2}-m_{\mathrm{res}}^{2}\right)^{2}+M^{2} \gamma_{\mathrm{bw}}^{2}} .
\end{aligned}
$$

Note that the normalization condition

$$
\int_{m_{\text {th }}}^{\infty} \frac{d M}{2 \pi} B_{\text {res }}(M)=1
$$

is satisfied provided that

$$
\delta_{\text {res }}(\infty)-\delta_{\text {res }}\left(m_{\text {th }}\right)=\pi
$$

for a threshold energy $m_{\mathrm{th}}$.

Furthermore, when the empirical phase shift from a partial wave analysis (PWA) of the scattering experiment is used for the calculation of $B(M)$, both resonant and nonresonant interactions are correctly incorporated, and the result becomes insensitive to the choice of parameters in an individual model.

As energy increases, new interaction channels open and the scattering becomes inelastic. The prescription of Eq. (13) remains valid, but the $S$ matrix should now be formally understood as a matrix acting in the open-channel space, i.e.,

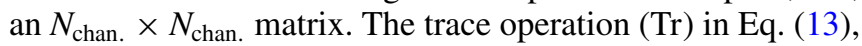
originated from the thermal trace in constructing the partition function, enforces a summation over the $N_{\text {chan. }}$ channels. The effective spectral function describing a multichannel system reads $[22,31,68]$

$$
B(M)=\sum_{a=1}^{N_{\text {chan. }}} B_{a}(M),
$$

where

$$
B_{a}(M)=\frac{1}{2} \operatorname{Im}\left[S^{-1} \frac{d}{d M} S-\left(\frac{d}{d M} S^{-1}\right) S\right]_{a a} .
$$

Here, $[\ldots]_{a a}$ means the $a$ th diagonal matrix element.

The channel-specific spectral function $B_{a}(M)$ describes the (energy-dependent) component of the full $B(M)$ when projected into a specific interaction channel $a$. It generalizes the standard prescription of a branching ratio $[\operatorname{Br}(X \rightarrow a)]$ in Eq. (4) and reflects the energy dependence from resonant and nonresonant interactions. The channel yield, e.g., from a resonance decaying into multiple final states, can be readily computed via

$$
n_{a}(T)=\int_{m_{\text {th }}}^{\infty} \frac{d M}{2 \pi} B_{a}(M) n^{(0)}(T, M),
$$

where $n^{(0)}$ is the ideal gas formula for the particle density.

In this study we focus on computing the hadron yields of protons and $\Lambda+\Sigma^{0}$ baryons. For the protons, we follow previous studies and employ the empirical phase shifts from the GWU/SAID PWA on the $\pi N$ scattering [69]. In addition, we implement a $\pi \pi N$ background contribution based on the LQCD computation of the baryon charge-susceptibility $\chi_{B Q}$. For the strange $(|S|=1)$ baryon system, we employ an existing coupled-channel model involving $\bar{K} N, \pi \Lambda$, and $\pi \Sigma$ interactions. The hyperon model allows to calculate not only the thermal yields of strange baryons, $\Lambda$ 's and $\Sigma$ 's, but also to quantify the additional contribution of protons from the strange baryons. We emphasize that the $S$-matrix scheme for $|S|=1$ hyperons is based on Ref. [70] and that the same scheme was used to compute the corresponding contribution to $\chi_{B S}$ in [22]. No extra tuning was done in the $|S|=1$ sector in this calculation.

\section{A. Composition of hadron yields}

Hadron yields predicted by a thermal model can be generally separated into two parts: a purely thermal yield determined by the freezeout parameters $T_{f}$ and $\vec{\mu}_{f}$, and a contribution which describes the multiparticle interaction involving the hadron species under study. The latter can be further classified according to the conserved quantum numbers of the interaction channel. For example the total proton yield can be written as

$$
\langle p\rangle=\langle p\rangle_{\mathrm{th}}+\langle p\rangle_{N^{*}}+\langle p\rangle_{\Delta}+\langle p\rangle_{\bar{K} N}+\ldots,
$$

where the notions $N^{*}, \Delta$, etc., stand for the general quantum numbers $(B=1 ; I=1 / 2,3 / 2)$ rather than referring to a specific resonance state.

The proton yield from a given interaction channel can be related to its thermal abundance. A further simplification enters when we focus on the freezeout conditions for describing hadron production in central nucleus-nucleus collisions at LHC energies: the freezeout chemical potentials are practically zero, and by isospin symmetry all isospin charge states contribute equally, giving

$$
\begin{aligned}
\langle p\rangle_{N^{*}} & =\frac{2}{3}\left\langle N_{Q=0}^{*}\right\rangle+\frac{1}{3}\left\langle N_{Q=1}^{*}\right\rangle \\
& \approx \frac{1}{2}\left\langle N^{*}\right\rangle,
\end{aligned}
$$

where $\left\langle N^{*}\right\rangle=\left\langle N_{Q=0}^{*}\right\rangle+\left\langle N_{Q=1}^{*}\right\rangle$ is the total thermal yield of $N^{*}$. The analysis proceeds similarly for protons emanating from $\Delta$ 's and other channels composed of a single nucleon.

We also compute the proton yields coming from the $(|S|=1)$ strange baryons system. The relevant channels are given by entries $(1,6,7,8,11-14)$ in Table I. In particular, quasi-two-body states like $\pi \Lambda(1520)$ can contribute via the channel weights $B_{13,14}$, after further multiplication by an inherent branching ratio $(\approx 45 \%$ to protons) of the resonance $\Lambda(1520) .{ }^{1}$ However, the dominant contribution comes from the $\bar{K} N\left(B_{1}\right)$ channel, where no further manipulation is required. The magnitude of $\langle p\rangle_{\text {hyp. }}$ from all hyperon channels is consistent with the HRG estimate based on branching ratios, and amounts to $\approx 6 \%$ of the total proton yield at $T=$ 156.5 MeV.

In Fig. 2 we show the proton density (normalized to $T^{3}$ ) as a function of temperature. As noted in previous studies, implementing the essential features of the empirical $\pi N$ scattering, i.e., the widths of resonances and the nonresonant

\footnotetext{
${ }^{1}$ Generally, channels involving $\Sigma(1385)$ and $\Lambda(1520)$ in the final states are multiplied by an inherent branching ratios, $(87,12,0) \%$ and $(10,43,45) \%$ respectively, when computing the yields of $(\Lambda$, $\Sigma$, protons).
} 

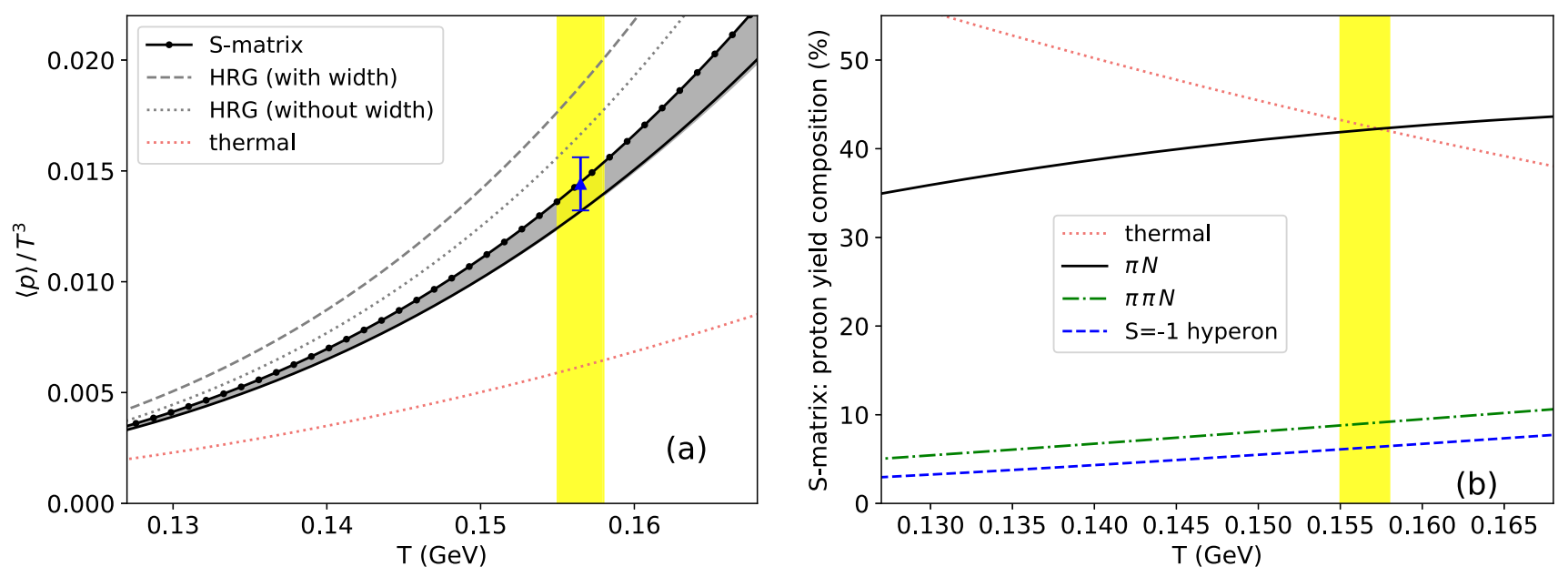

FIG. 2. The proton yield density (left) and a composition analysis (right) computed within the $S$-matrix formulation. The corresponding results from the HRG scheme, implemented with and without resonance widths, are also shown. For the $S$-matrix prediction: solid black points correspond to including the full list of interactions, and the black line represents the partial result accounting only for the elastic and quasielastic scatterings, i.e., without the $\pi \pi N$ background contribution constrained by the LQCD result for $\chi_{B Q}$. The blue triangle depicts the proton yield density calculated from the proton yield data measured by the ALICE collaboration for the most central $\mathrm{Pb}$ - $\mathrm{Pb}$ collisions (at 2.76 $\mathrm{TeV}$ ) and the fireball volumes in Eq. (23). The error bar represents only the experimental errors of the yield data.

interactions, via the $S$-matrix formalism leads to a reduction of the proton yield relative to the HRG baseline. Including the protons from strong decays of $|S|=1$ hyperons does not alter this conclusion.

An estimate of the contribution by the $\pi \pi N$ background, based on LQCD results on the baryon-charge susceptibility $\chi_{B O}$, is also displayed. This gives $\approx 8.6 \%$ of the total proton yield at the LHC freezeout conditions. Note that it is an independent source of protons compared to the ones from the hyperons as the latter do not contribute to $\chi_{B Q}$ in an isospinsymmetric system [21]. A dissection of the composition of proton yield in the full $S$-matrix scheme is shown in Fig. 2 (right).

We perform an analogous study for the $\Lambda+\Sigma^{0}$ yields based on the multichannel hyperon model. The result is shown in Fig. 3(left). Here, it is convenient to classify the interaction channels into three groups:

Group I: elastic scatterings of elementary (ground state) hadrons (channels $1-5$ in Table I).

Group II: quasi-two-body states involving the resonances $K^{*}(892), \Sigma(1385)$, and $\Delta(1232)$ (channels 6-14 in Table I).

Group III: dummy channels that collectively account for the missing inelasticity arising from channels not included explicitly (channels 15-16 in Table I).

Groups II and III should be formally understood as an effective treatment of three-body final states within the framework of an isobar decomposition, which is also compatible with the notion of effective elementary in thermal state counting $[71,72]$.

The $16 \times 16 S$ matrix is computed for the following partial waves: for $I=0: S_{01}, P_{01}, P_{03}, D_{03}, D_{05}, F_{05}, F_{07}$, and $G_{07}$ and for $I=1: S_{11}, P_{11}, P_{13}, D_{13}, D_{15}, F_{15}, F_{17}$, and $G_{17}$ cases. The subscripts specify the quantum numbers $(I, 2 \times J)$, where $I$ is isospin and $J$ is total spin. These are included in computing the thermal yields of protons and hyperons.
Most channels in the Groups I and II have single-energy partial-wave data to fit, see [70] for details. To distinguish between the different levels of approximation, we employ the following convention to present the results: The black points correspond to the full $S$-matrix scheme, including the full list of interactions. The solid black lines are partial results accounting only for the elastic and quasielastic scatterings, i.e., Groups I and II. The shaded region (in gray) shows the values spanned from including only Group I to the full list of interactions.

Unlike in the case of protons, the hyperon model predicts a higher $\Lambda+\Sigma^{0}$ yield than the HRG baseline. The $S$-matrix approach entails the proper treatment of resonances and naturally incorporates some additional hyperon states beyond the listing of the three- and four-star PDG states [23]. A table of the resonances identified in the PWA model is available from [22]. The need for a stronger interaction strength has already been indicated by an analysis of the LQCD results of $\chi_{B S}$ [20].

A composition analysis of the $\Lambda+\Sigma^{0}$ yield is shown in Fig. 3(right). The $S$-matrix scheme predicts that a substantial fraction of the hyperons comes from the quasi-two-body states and the three-body backgrounds. The latter, constrained only by unitarity, represents the major uncertainty in the model. Theoretical analysis of the amplitudes beyond the elastic, two-body limit is, as a rule, much more involved. In particular, the proper treatment of these channels as genuine three-body states remains a challenging task, and is currently under active development [73-76]. To make progress we attempt to extract a phenomenological estimate of the densities based on measured hadron yields and the fireball volume from a thermal model.

\section{B. Estimation of hadron densities}

As discussed, the LQCD results on $\chi_{B S}$ support an enhancement in the thermal yields of hyperons, while those on 

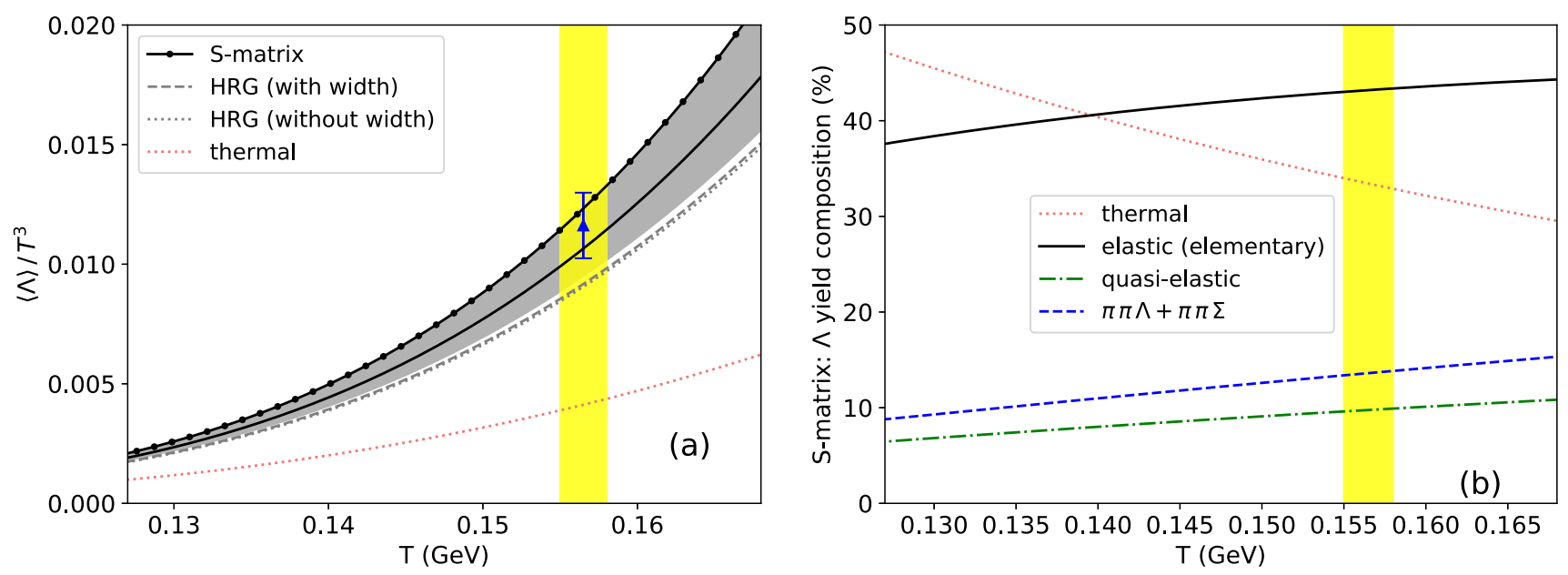

FIG. 3. Similar to Fig. 2 but for the $\Lambda+\Sigma^{0}$-baryon yield density. The shaded region (in gray) shows the range of predictions by the $S$-matrix scheme at different levels of approximation: from accounting only for the elastic scattering of elementary states (bottom), adding the quasielastic scatterings (black solid line), to including the full list of interactions (black points). See Table I for the list of interaction channels in the multichannel hyperon model.

the $\chi_{B Q}$ suggests a suppression of thermal proton yield. It is then of interest to investigate whether a particle yield analysis by a thermal model would support these claims.

A key quantity to extract from a thermal model in the GC ensemble at a given temperature is the volume of the fireball. See Eq. (23) and the discussion. The volume is the same for all hadron species, and is obtained from a global fit to all hadrons. Clearly, the volume thus obtained is not free from the influence of densities of protons and $\Lambda+\Sigma^{0}$ baryons, the latter we are trying to estimate. However, the value is most strongly determined by the most abundant hadrons in the fireball, i.e., the pions. Thus, constructing the hadron densities based on measured yields and the fireball volume gives a consistent estimate of the hadron densities by a thermal model. With the volumes constructed for the most central $\mathrm{Pb}-\mathrm{Pb}$ collisions (at $2.76 \mathrm{TeV}$, averaged over the three data points with the highest multiplicities), the estimate for hadron densities are computed and are shown as blue triangles in Figs. 2 and 3.

For protons the estimate agrees with the full $S$-matrix result. Note the different origins of the error on the estimate and the gray band: the former is due to the experimental error of the measured proton yield, the latter represents the effect of including or excluding the contribution from the $\pi \pi N$ backgrounds. For the hyperons, the density estimate supports the trend predicted by the $S$-matrix approach: an increase in the hyperon yields compared to the HRG baseline. The estimate substantiates the need for including quasi-two-body states [up to the black line in Fig. 3(left)] and in addition part of the three-body unitarity backgrounds. The remaining discrepancy may be resolved by including other channels such as $K \Xi$ (and other multi-strange states), and an improved treatment of the three-body states.

It is also possible to construct observables that are independent of the fireball volume. An interesting quantity to consider is the ratio of the yields of protons to that of $\Lambda+\Sigma^{0}$ baryons. As discussed, the HRG scheme tends to overestimate the numerator (more so for the scheme with resonance widths) while underestimating the denominator. This results in a much larger value than the prediction of the $S$-matrix scheme as shown in Fig. 4. Evidently, the result obtained from the measured hadron yields favors the latter, demonstrating the robustness of the S-matrix scheme. Further thermal model analysis of the measured particle yields will be presented in the next section.

\section{MODEL COMPARISON WITH ALICE DATA}

In the GC ensemble with $\vec{\mu}=\overrightarrow{0}$ and for constant temperature the yields of all particles per volume should be clearly independent of $d N_{\mathrm{ch}} / d \eta$. On the other hand, from the previous section, it is clear that the density of charged particles in the $\mathrm{C}$ ensemble exhibits a nonlinear dependence on volume parameters. Consequently, in a thermal model any dependence of charged particle densities on $V$ can be a strong

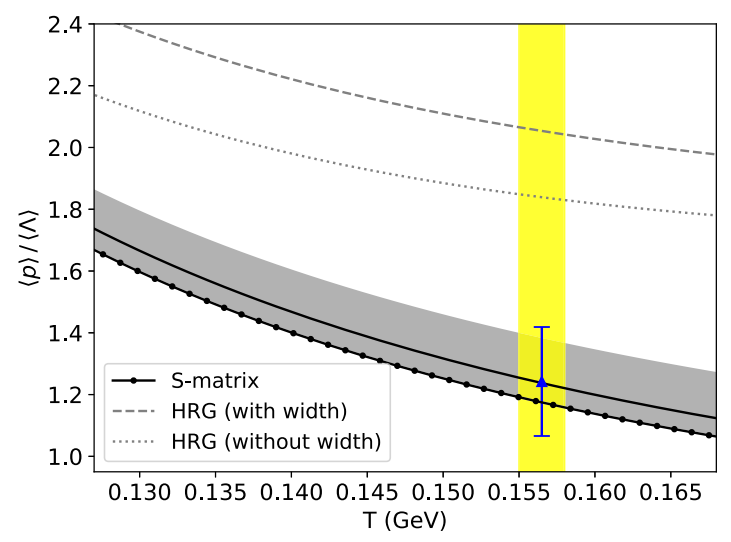

FIG. 4. The ratio of the proton yield to that of $\Lambda+\Sigma^{0}$ baryons. The predictions of the $S$-matrix scheme at various levels of approximation (see text for conventions) and those from the HRG scheme (with and without width) are shown. The blue triangle depicts the experimental value measured by the ALICE collaboration for the most central $\mathrm{Pb}-\mathrm{Pb}$ collisions (at $2.76 \mathrm{TeV}$ ). 
indication of possible corrections due to exact conservation of quantum numbers. Indeed, in the context of strangeness production at the LHC, already from a previous analysis [77] it was clear that the canonical ensemble with exact strangeness conservation (CSE) is the best ensemble for describing the low multiplicity classes in $p$ - $p$ collisions at the LHC. Furthermore, the CSE was shown to connect better to the high multiplicity classes than the canonical ensemble with exact baryon, strangeness and charge conservation [77]. Thus, in the following, we focus on only exact strangeness conservation and analyze data obtained by the ALICE collaboration on (multi)strange particle multiplicity in $p$ - $p$ collisions at $7 \mathrm{TeV}$ [52] as well as $13 \mathrm{TeV}$ [61], in $p$ - $\mathrm{Pb}$ collisions at 5.02 $\mathrm{TeV}[8,53]$ and in $\mathrm{Pb}-\mathrm{Pb}$ collisions at 2.76 $\mathrm{TeV}$ [54-56]. The thermal model calculations are done using the latest version of THERMUS [34,78] which is further extended here to account for a more complete description of hadron interactions within the $S$-matrix approach. We consider the $S$-matrix corrections to proton and hyperon yields as introduced in the previous section.

As a first step, we made a fit for each multiplicity bin by keeping the number of parameters to a minimum. For the temperature we are guided by the results from LQCD [12] and the recent HRG model analysis of ALICE data for central $\mathrm{Pb}-\mathrm{Pb}$ collisions $[1,5]$ and allow only two choices: $T_{f}=156.5 \mathrm{MeV}$ and $T_{f}=160 \mathrm{MeV}$, to identify $T$ variation of particle yields. For the strangeness suppression factor $\gamma_{s}$ we work with the value $\gamma_{s}=1$ as motivated by fits in central $\mathrm{Pb}-\mathrm{Pb}$ collisions, this is in contrast to $[7,79]$ where substantial deviations have been proposed. The chemical potentials due to conservation of baryon number and electric charge are being set to zero as in the energy region of interest at the LHC, particle-antiparticle symmetry is observed with a good degree of accuracy. Thus, in the SCE only two parameters remain, the volume of the system in the experimental acceptance $V_{A}$ and the canonical volume $V_{C}$ which quantifies the range of exact strangeness conservation. An independent determination of the $V_{C}$ parameter remains an open issue.

For large multiplicities we have found that $V_{A} \simeq V_{C}$, therefore we first put them equal for all multiplicities. In Fig. 5(left) we show the yields of hadrons calculated in the SCE for different charged particle multiplicities $d N_{\mathrm{ch}} / d \eta$ at fixed temperature and for a single volume $V=V_{A} \simeq V_{C}$. It is to be noted that, the charged particle multiplicity is measured by ALICE collaboration in the pseudorapidity range $-0.5<\eta<$ 0.5 , while the particle yields are measured in a rapidity interval $-0.9<y<0.9$.

The SCE model is seen in Fig. 5(left) to capture basic properties of hadron yields data already with a single volume parameter. For large $d N_{\mathrm{ch}} / d \eta>100$ all hadron yields, as well as extracted $V$, depend linearly on charged particle rapidity density. However, for lower $d N_{\mathrm{ch}} / d \eta$ this dependence is clearly non-linear for strange particles due to strangeness canonical suppression which increases with the strangeness content of particles. On the quantitative level, however, one can see in Fig. 5(left), that using a single volume, leads to too much suppression at small charged particle multiplicities, particularly for $S=-2$ and $S=-3$ baryons. This result is consistent with the previous observation, that a single volume

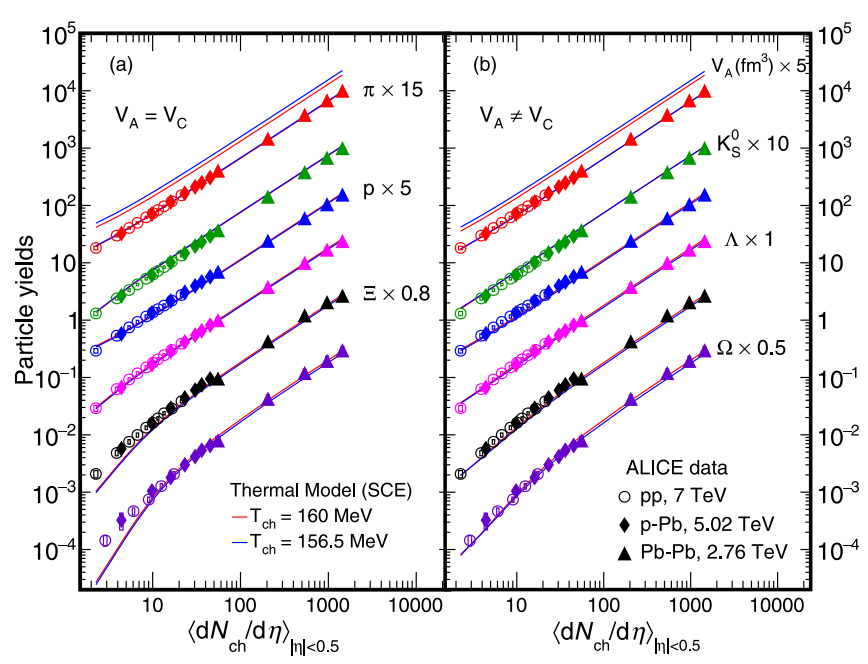

FIG. 5. Left: Yields for $V_{A}=V_{C}$. Right: Yields for $V_{A} \neq V_{C}$, The two top lines are the fitted volumes $V_{A}(\times 5)$ in $\mathrm{fm}^{3}$. The particle yields are indicated in the right panel together with the multiplicative factor used to separate the yields. The solid blue lines have been calculated for $T=156.5 \mathrm{MeV}$ while the solid red lines have been calculated for $T=160 \mathrm{MeV}$. The values of the volumes used have been parametrized empirically in Eqs. (23) and (24).

canonical model implies too strong strangeness suppression in low multiplicity events [53,62,64].

In general, strangeness conservation relates to the full phase-space whereas particle yields are measured in some acceptance window. Thus, the strangeness canonical volume parameter $V_{C}$ can be larger than the fireball volume $V_{A}$, restricted to a given acceptance. To quantify ALICE data we have performed the SCE model fit to data with two independent volume parameters as shown in Fig. 5(right). The resulting yields exhibit much better agreement with data by decreasing strangeness suppression at lower multiplicities due to larger value of $V_{C}$ than $V_{A}$.

The fitted volume parameters are shown in Fig. 6 for $T=$ $160 \mathrm{MeV}$. Two features are to be noted in this figure. First of all the overall volume $V_{A}$ can be determined fairly accurately and increases linearly with the charged-particle multiplicity. This strongly supports that the yields are directly proportional to the volume of the fireball and agrees with one of the basic ingredients of the thermal model. Second, the canonical volume $V_{C}$ differs from $V_{A}$, however the difference is not so well determined at larger $d N_{\mathrm{ch}} / d \eta$ as it appears in a ratio of Bessel functions which is already near to its asymptotic value. For small multiplicities, however, the value of $V_{C}$ is clearly larger than $V_{A}$ leading to a reduced suppression of strange-particle yields. The fits to $V_{A}$ and $V_{C}$ were made for each multiplicity bin and can be well parametrized as linear functions of charged particle multiplicity:

$$
\begin{aligned}
& V_{A}=1.27+2.58 \times \frac{d N_{\mathrm{ch}}}{d \eta} \text { for } T=160 \mathrm{MeV}, \\
& V_{A}=1.55+3.02 \times \frac{d N_{\mathrm{ch}}}{d \eta} \text { for } T=156.5 \mathrm{MeV} .
\end{aligned}
$$




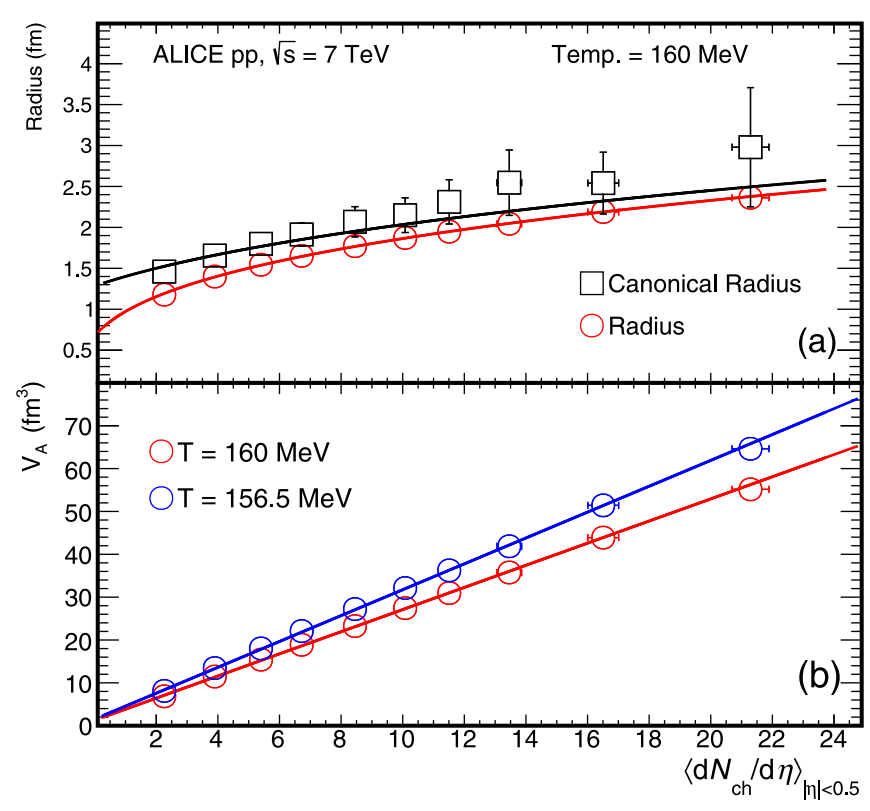

FIG. 6. The upper panel shows the radius (red points) as extracted from fits to $p$ - $p$ collisions at $7 \mathrm{TeV}$ as a function of the charged hadron multiplicity in midrapidity region using the SCE model with $T=160 \mathrm{MeV}$ and $\gamma_{s}=1$. The black points in the upper figure show the values of the canonical radius. The lower panel shows the volume $V_{A}=4 / 3 \pi R^{3}$ as a function of the charged particle multiplicity for two values of the chemical freezeout temperature, red points were obtained from $T=160 \mathrm{MeV}$, blue points are for $T=156.5 \mathrm{MeV}$.

The volume is slightly larger for $T=156.5 \mathrm{MeV}$ than for $T=160 \mathrm{MeV}$ so as to compensate for the smaller particle densities. A possible form for the canonical volume is given by

$$
\begin{aligned}
& V_{C}=8.87+2.64 \times \frac{d N_{\mathrm{ch}}}{d \eta} \text { for } T=160 \mathrm{MeV} \\
& V_{C}=12.32+3.02 \times \frac{d N_{\mathrm{ch}}}{d \eta} \text { for } T=156.5 \mathrm{MeV}
\end{aligned}
$$

These parametrizations are shown in Fig. 6 as lines and have been used in all our model comparisons to data. All numbers in Eqs. (23) and (24) are in units of $\left[\mathrm{fm}^{3}\right]$. We emphasize that the parametrizations of the volumes given in Eqs. (23) and (24) are purely empirical. It is indeed interesting to see a linear dependence (in $d N / d \eta$ ) of the acceptance volume, which to a good accuracy is also independent of the collision system.

To appreciate the quality of the SCE model description of ALICE data illustrated in Fig. 5(right), we show in Fig. 7 the ratio of data and the model results. It can be seen that the model prediction agrees quite well with the data up to two standard deviations for all $d N_{\mathrm{ch}} / d \eta$. The data on pion yields are always slightly above the calculated points while the kaons are always below. This has implications for the kaon to pion ratio discussed further below. This illustrates the pitfalls of showing ratios in the thermal model. It is better to compare directly yields.

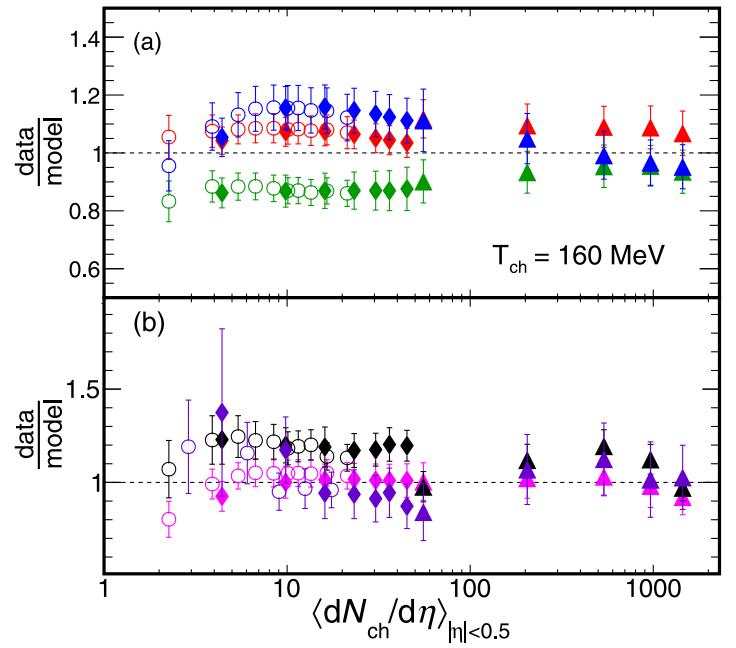

FIG. 7. Data relative to model calculations. The upper panel shows the protons in blue, the pions in red, the kaons in green. The lower panel shows the $\Xi$ in black, the $\Lambda$ in magenta, and the $\Omega$ in purple.

The strangeness suppression effect and its CSE model description are particularly transparent when removing an overall linear dependence of particle yields on the fireball volume $V_{A}$. This isachieved by plotting the ratio of strange particle and pion yields, as shown in Fig. 8. This ratio has been discussed prominently by the ALICE collaboration [52] where a comparison with other model calculations was presented. The SCE model introduced here compares very favorably to the ones discussed in [52]. The underestimation of the pion yield is responsible for the larger discrepancy in the kaon to pion ratio as seen in Fig. 7.

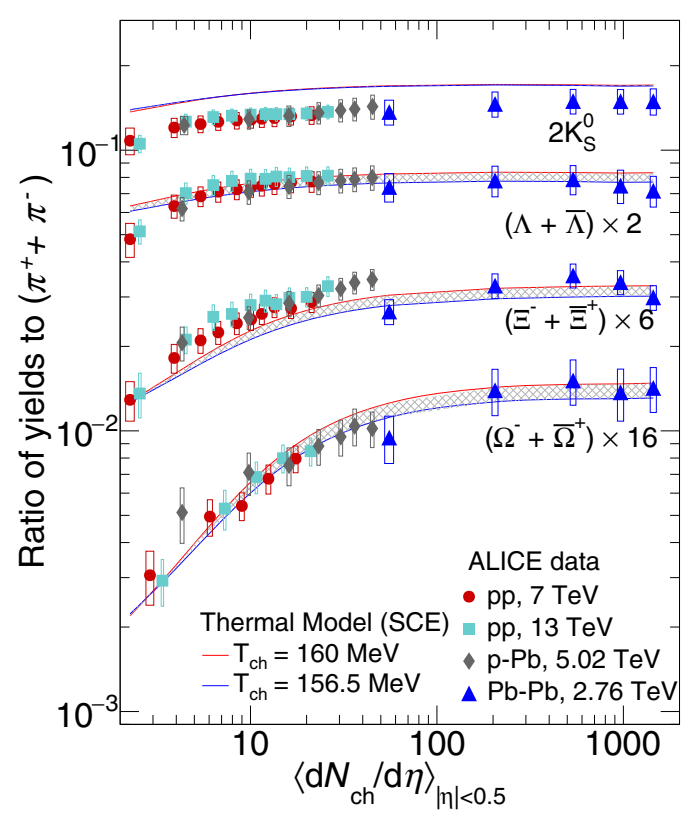

FIG. 8. Ratios of yields of strange particles to pions versus charged particle multiplicity. The SCE model results were obtained using $V_{A} \neq V_{C}$ as explained in the text. 

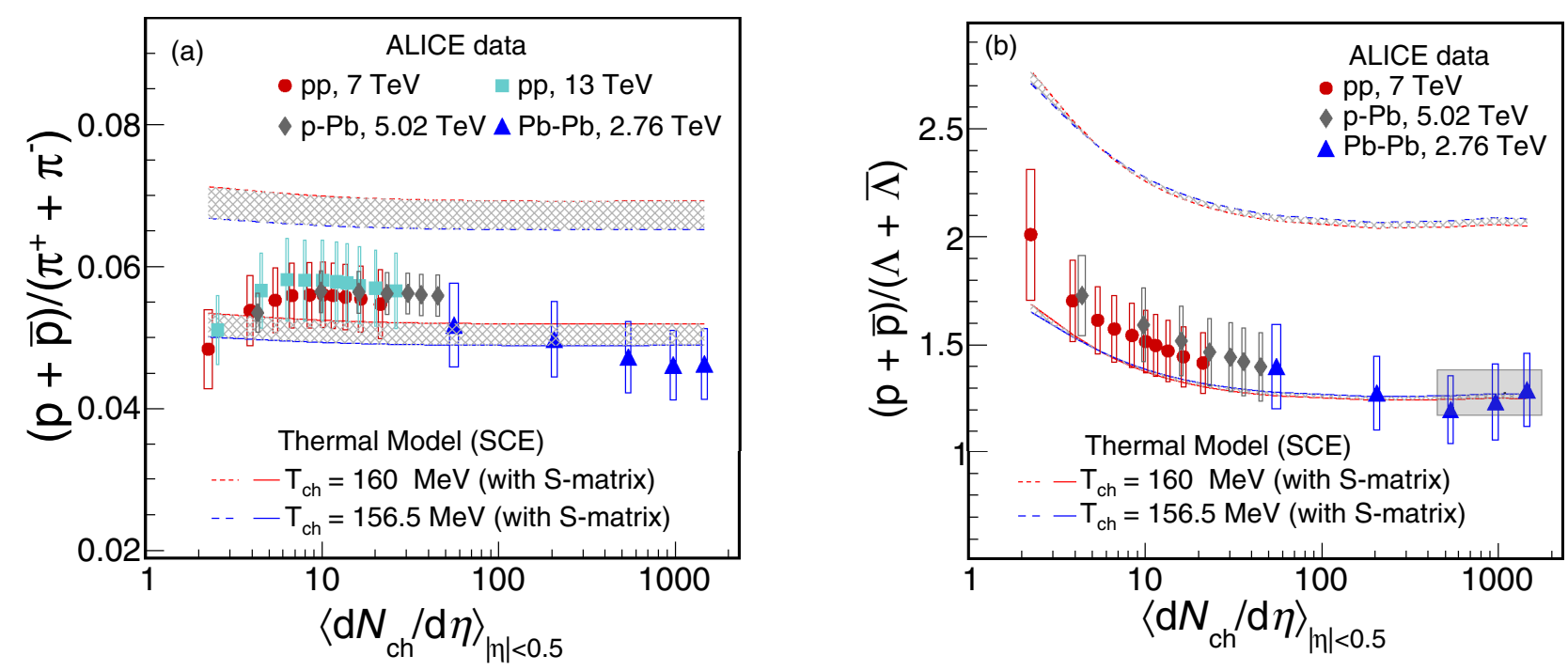

FIG. 9. Left: Proton to pion ratio showing the importance of $S$-matrix corrections. Right: Proton to $\Lambda$ ratio showing the importance of $S$-matrix corrections. The lower lines take into account the $S$-matrix corrections, the upper lines are obtained without those corrections. The shaded region (in gray) shows the theoretical predictions spanned by different stages of the $S$-matrix improvement: from including only elastic scatterings of ground state hadrons (upper limit) to the full list of interactions (lower limit). See text for details.

The importance of the $S$-matrix description of proton and hyperon yields can be directly verified with data from the central $\mathrm{Pb}-\mathrm{Pb}$ collisions by comparing measured $p / \Lambda$ ratio with the $S$-matrix results shown in Fig. 4 . It is seen in this figure, that data are indeed well consistent with $S$-matrix predictions at $T_{f} \simeq T_{c}$, indicating that particles are produced at the phase boundary. Furthermore, such comparison can also be done for densities of protons and hyperons as discuss in the context of Figs. 2 and 3 by using yields data and the fitted fireball volume for the central $\mathrm{Pb}-\mathrm{Pb}$ collisions.

To justify the $S$-matrix results for different charged particle densities we show in Fig. 9 the $p / \pi$ (left) and $p / \Lambda$ (right) ratios as functions of $d N_{\mathrm{ch}} / d \eta$. Both ratios are very sensitive to the $S$-matrix corrections which amount to a reduction of proton yield by a factor of 0.75 and a 1.24 enhancement of the $\Lambda$ yield. The excellent agreement of data and the $S$-matrix values that have been already discussed for most central collisions are also verified for lower charged particle multiplicities. The $p / \Lambda$ ratio is increasing with decreasing $d N_{\mathrm{ch}} / d \eta$ with a strength which is well consistent with the SCE model. The thermal model without $S$-matrix corrections exhibits large deviations from the $p / \Lambda$ ratio data. The $p / \pi$ is nearly multiplicity independent since the SCE corrections to this ratio are small. Nevertheless, the $p / \pi$ ratio with the $S$-matrix corrections is well consistent within errors with the data at all $d N_{\mathrm{ch}} / d \eta$.

In Fig. 10 we show ratios of strange and multi-strange particles compared to the $\Lambda$ yield. The model results for the $\Omega / \Lambda$ ratio describe data very accurately while the two other ratios, $K_{S}^{0} / \Lambda$ and $\Xi / \Lambda$, are again within one standard deviation. It is interesting to note, that in the SCE model the $K_{S}^{0} / \Lambda$ ratio is independent of $d N_{\mathrm{ch}} / d \eta$. This is because the canonical suppression factor is the same for all $S= \pm 1$ mesons and baryons. The results shown in Figs. 9 and 10 provide further evidence that the $S$-matrix description of interactions in the proton and hyperon channels, as well as, that the strangeness suppression due to exact and global strangeness conservation, are justified by data from the ALICE collaboration.

\section{SUMMARY AND CONCLUSIONS}

We have studied the influence of global strangeness quantum number conservation on strangeness production in

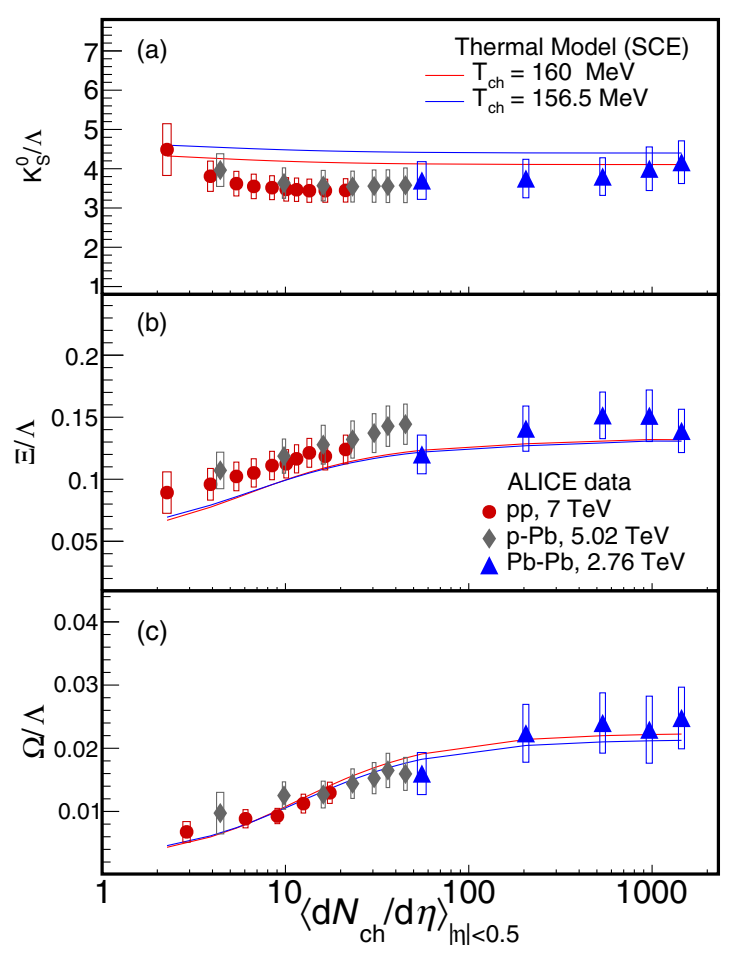

FIG. 10. Ratios of $K_{S}^{0}$ (upper panel), $\Xi$ (middle panel), and $\Omega$ (lower panel) to $\Lambda$ yields. All lines were calculated taking into account $S$-matrix corrections as discussed in the text. 
heavy-ion and elementary collisions in a given acceptance region, accounting for the strangeness neutrality condition formulated in the hadron resonance gas (HRG) model in the canonical ensemble. We have focused on (multi)strange baryon production yields in $p-p, p-A$, and $A-A$ collisions at the LHC energies and their behavior with charged-particle multiplicity at mid-rapidity, as measured by the ALICE collaboration at CERN.

To this end, the HRG model is augmented with the $S$ matrix corrections to the yields of protons and hyperons. The $S$-matrix calculation is based on the empirical phase shifts of $\pi N$ scattering, an estimate of the $\pi \pi N$ background constrained by lattice QCD results of baryon-charge susceptibility, and an existing coupled-channel model describing the $|S|=1$ strange baryons. It is demonstrated that an accurate description of the widths of resonances and the nonresonant interactions in a thermal model leads to a reduction of the proton yield relative to the HRG baseline (by $\approx 25 \%$ ). Including the protons from strong decays of $|S|=1$ hyperons, which constitute $\approx 6 \%$ of the total yields, does not alter this conclusion. Such a reduction is also crucial for resolving the proton anomaly in the LHC data. For the hyperons, the $S$-matrix scheme predicts an increase in the $\Lambda+\Sigma^{0}$ yields relative to the HRG baseline by $\approx 23 \%$. This is consistent with the data from the ALICE collaboration. Furthermore, the $S$-matrix prediction on the ratio of yields of proton to $\Lambda+\Sigma^{0}$ is in good agreement with the measured values by the ALICE collaboration in $\mathrm{Pb}-\mathrm{Pb}$ collisions in the events with the largest multiplicities $\left(d N_{\mathrm{ch}} / d \eta\right)$. The evolution of (multi)strange baryons to $\Lambda$ yields with $d N_{\mathrm{ch}} / d \eta$ calculated in the present thermal model in the $\mathrm{C}$ ensemble follows the measured values within two standard deviations.

Comparing these experimental findings alongside the thermal model predictions (including the $S$-matrix corrections), it is evident that an accurate treatment of two-body scatterings and in addition the three-body interactions, at least those captured within the quasi-two-body framework, is necessary for a satisfactory description of data. A more realistic theoretical treatment of the three-body interactions may resolve the remaining background contribution.

A good description was obtained for the variation of the strangeness content in the final state as a function of the number of charged hadrons at midrapidity with the same freezeout temperature $T_{f} \sim 156.5 \mathrm{MeV}$ as calculated previously from data in the most central $\mathrm{Pb}-\mathrm{Pb}$ collisions. This lends further support that at LHC energies and independently of colliding system, the freezeout temperature coincides with the chiral crossover as calculated in LQCD.

We have argued that the observed behavior of hadron yields at the LHC with charged particle multiplicity can be explained naturally in the thermal model. The temperature is linked to the collision energy and is independent of the colliding system. The fireball volume parameter at mid-rapidity was found to scale linearly with $d N_{\mathrm{ch}} / d \eta$. The observed increasing suppression of strange hadron yields with decreasing $d N_{\mathrm{ch}} / d \eta$ and its dependence on their strangeness content, was found to be qualitatively consistent with predictions of the $\mathrm{HRG}$ model formulated in the strangeness canonical ensemble. An exact conservation of strangeness is to be imposed in the full phase-space rather than in the experimental acceptance at midrapidity. Consequently, the correlation volume parameter where strangeness is exactly conserved was found to be larger than the fireball volume at midrapidity.

The $S$-matrix formulation of statistical mechanics accurately describes the measured hadron yields and supports the interpretation of the LQCD results. This encourages its further adoption in analyzing the multistrange baryon sectors and the light mesons. The thermal yield data can guide future $S$-matrix modeling, which allows a more reliable assessment of the relevance of, for example, the extra quark model states [80] in $\chi_{B S}$ and in higher order fluctuations. Also, many Fock states considered in the current scheme contain multipion components. It is thus pivotal to investigate their influence on the thermal production of pions and kaons. This requires an extensive analysis involving also the purely mesonic sector [81], and will be explored in a future study [82].

\section{ACKNOWLEDGMENTS}

P.M.L. and K.R. acknowledge the support by the Polish National Science Center (NCN) under Opus Grant no. 2018/31/B/ST2/01663. K.R. also acknowledges partial support of the Polish Ministry of Science and Higher Education, and fruitful discussions with A. Andronic, P. Braun-Munzinger, B. Friman, A. Rustamov, and J. Stachel. N.S. acknowledges the support of SERB Ramanujan Fellowship (D.O. No. SB/S2/RJN-084/2015) of the Department of Science and Technology, Government of India.
[1] A. Andronic, P. Braun-Munzinger, K. Redlich, and J. Stachel, Nature 561, 321 (2018).

[2] J. Stachel, A. Andronic, P. Braun-Munzinger, and K. Redlich, J. Phys. Conf. Ser. 509, 012019 (2014).

[3] A. Andronic, Int. J. Mod. Phys. A 29, 1430047 (2014).

[4] F. Becattini, P. Castorina, A. Milov, and H. Satz, Eur. Phys. J. C 66, 377 (2010).

[5] A. Andronic, P. Braun-Munzinger, B. Friman, P. M. Lo, K. Redlich, and J. Stachel, Phys. Lett. B 792, 304 (2019).

[6] S. Chatterjee, S. Das, L. Kumar, D. Mishra, B. Mohanty, R. Sahoo, and N. Sharma, Adv. High Energy Phys. 2015, 349013 (2015).
[7] S. Das, D. Mishra, S. Chatterjee, and B. Mohanty, Phys. Rev. C 95, 014912 (2017).

[8] B. B. Abelev et al. (ALICE Collaboration), Phys. Lett. B 728, 25 (2014).

[9] J. Cleymans and K. Redlich, Phys. Rev. C 60, 054908 (1999).

[10] A. Andronic, P. Braun-Munzinger, M. K. Köhler, K. Redlich, and J. Stachel, Phys. Lett. B 797, 134836 (2019).

[11] A. Andronic, P. Braun-Munzinger, M. Köhler, and J. Stachel, Nucl. Phys. A 982, 759 (2019).

[12] A. Bazavov et al. (HotQCD Collaboration), Phys. Lett. B 795, 15 (2019). 
[13] S. Borsanyi, Z. Fodor, J. N. Guenther, R. Kara, S. D. Katz, P. Parotto, A. Pasztor, C. Ratti, and K. K. Szabo, Phys. Rev. Lett. 125, 052001 (2020).

[14] P. Braun-Munzinger, B. Friman, F. Karsch, K. Redlich, and V. Skokov, Phys. Rev. C 84, 064911 (2011).

[15] P. Braun-Munzinger, B. Friman, F. Karsch, K. Redlich, and V. Skokov, Nucl. Phys. A 880, 48 (2012).

[16] P. Braun-Munzinger, A. Kalweit, K. Redlich, and J. Stachel, Phys. Lett. B 747, 292 (2015).

[17] F. Becattini, J. Steinheimer, R. Stock, and M. Bleicher, Phys. Lett. B 764, 241 (2017).

[18] A. Bazavov et al., Phys. Rev. D 95, 054504 (2017).

[19] J. Noronha-Hostler, P. Parotto, C. Ratti, and J. M. Stafford, Phys. Rev. C 100, 064910 (2019).

[20] A. Bazavov et al., Phys. Rev. Lett. 113, 072001 (2014).

[21] P. M. Lo, B. Friman, K. Redlich, and C. Sasaki, Phys. Lett. B 778, 454 (2018).

[22] C. Fernández-Ramírez, P. M. Lo, and P. Petreczky, Phys. Rev. C 98, 044910 (2018).

[23] M. Tanabashi et al. (Particle Data Group), Phys. Rev. D 98, 030001 (2018).

[24] P. Alba, V. M. Sarti, J. Noronha-Hostler, P. Parotto, I. PortilloVazquez, C. Ratti, and J. M. Stafford, Phys. Rev. C 101, 054905 (2020).

[25] R. Dashen, S.-K. Ma, and H. J. Bernstein, Phys. Rev. 187, 345 (1969).

[26] R. Venugopalan and M. Prakash, Nucl. Phys. A 546, 718 (1992).

[27] W. Weinhold, B. Friman, and W. Norenberg, Phys. Lett. B 433, 236 (1998).

[28] A. Dash, S. Samanta, and B. Mohanty, Phys. Rev. C 99, 044919 (2019).

[29] A. Dash, S. Samanta, and B. Mohanty, Phys. Rev. C 97, 055208 (2018).

[30] F. Giacosa, V. Begun, and W. Broniowski, Acta Phys. Pol. Supp. 9, 213 (2016).

[31] P. M. Lo, Eur. Phys. J. C 77, 533 (2017).

[32] B. Friman, P. M. Lo, M. Marczenko, K. Redlich, and C. Sasaki, Phys. Rev. D 92, 074003 (2015).

[33] P. M. Lo, B. Friman, M. Marczenko, K. Redlich, and C. Sasaki, Phys. Rev. C 96, 015207 (2017).

[34] S. Wheaton, J. Cleymans, and M. Hauer, Comput. Phys. Commun. 180, 84 (2009).

[35] P. Braun-Munzinger, B. Friman, K. Redlich, A. Rustamov, and J. Stachel, arXiv:2007.02463 [nucl-th].

[36] A. Bzdak, V. Koch, and V. Skokov, Phys. Rev. C 87, 014901 (2013).

[37] P. Braun-Munzinger, A. Rustamov, and J. Stachel, Nucl. Phys. A 982, 307 (2019).

[38] R. Hagedorn, (1971), doi: 10.5170/CERN-1971-012.

[39] R. Hagedorn and K. Redlich, Z. Phys. C 27, 541 (1985).

[40] J. Cleymans, K. Redlich, and E. Suhonen, Z. Phys. C 51, 137 (1991).

[41] J. Cleymans, H. Oeschler, and K. Redlich, Phys. Rev. C 59, 1663 (1999).

[42] S. Hamieh, K. Redlich, and A. Tounsi, Phys. Lett. B 486, 61 (2000).

[43] C. M. Ko, V. Koch, Z.-W. Lin, K. Redlich, M. Stephanov, and X.-N. Wang, Phys. Rev. Lett. 86, 5438 (2001).

[44] P. Braun-Munzinger, K. Redlich, and J. Stachel, in QuarkGluon Plasma 3, edited by R. C. Hwa and X.-N. Wang,
Quark-Gluon Plasma (World Scientific, Singapore, 2003), pp. 491-599.

[45] V. V. Begun, M. Gaździcki, M. I. Gorenstein, and O. S. Zozulya, Phys. Rev. C 70, 034901 (2004).

[46] K. Redlich and A. Tounsi, Eur. Phys. J. C 24, 589 (2002).

[47] I. Kraus, J. Cleymans, H. Oeschler, and K. Redlich, Phys. Rev. C 79, 014901 (2009).

[48] N. Sharma, J. Cleymans, and L. Kumar, Eur. Phys. J. C 78, 288 (2018).

[49] A. Andronic, F. Beutler, P. Braun-Munzinger, K. Redlich, and J. Stachel, Phys. Lett. B 675, 312 (2009).

[50] F. Becattini, P. Castorina, J. Manninen, and H. Satz, Eur. Phys. J. C 56, 493 (2008).

[51] S. Acharya et al. (ALICE Collaboration), Eur. Phys. J. C 80, 693 (2020).

[52] J. Adam et al. (ALICE Collaboration), Nat. Phys. 13, 535 (2017).

[53] J. Adam et al. (ALICE Collaboration), Phys. Lett. B 758, 389 (2016).

[54] B. Abelev et al. (ALICE Collaboration), Phys. Rev. C 88, 044910 (2013).

[55] B. B. Abelev et al. (ALICE Collaboration), Phys. Rev. Lett. 111, 222301 (2013).

[56] B. B. Abelev et al. (ALICE Collaboration), Phys. Lett. B 728, 216 (2014); 734, 409 (2014).

[57] P. b. R. Fini et al. (WA97 Collaboration), J. Phys. G 27, 375 (2001).

[58] M. Aggarwal et al. (STAR Collaboration), Phys. Rev. C 83, 024901 (2011).

[59] L. Adamczyk et al. (STAR Collaboration), Phys. Rev. C 96, 044904 (2017).

[60] J. Cleymans, S. Kabana, I. Kraus, H. Oeschler, K. Redlich, and N. Sharma, Phys. Rev. C 84, 054916 (2011).

[61] S. Acharya et al. (ALICE Collaboration), Eur. Phys. J. C 80, 167 (2020).

[62] V. Vislavicius and A. Kalweit, arXiv:1610.03001 [nucl-ex].

[63] A. Kalweit, J. Phys. Conf. Ser. 779, 012021 (2017).

[64] J. Anielski, Ph.D. thesis, Westfälische Wilhelms-Universität, Münster, Germany, 2014.

[65] H. Satz, Acta Phys. Pol. Supp. 10, 711 (2017).

[66] P. Castorina and H. Satz, Int. J. Mod. Phys. E 23, 1450019 (2014).

[67] P. Braun-Munzinger, J. Cleymans, H. Oeschler, and K. Redlich, Nucl. Phys. A 697, 902 (2002).

[68] P. M. Lo, Phys. Rev. D 102, 034038 (2020).

[69] R. L. Workman, R. A. Arndt, W. J. Briscoe, M. W. Paris, and I. I. Strakovsky, Phys. Rev. C 86, 035202 (2012).

[70] C. Fernández-Ramírez, I. V. Danilkin, D. M. Manley, V. Mathieu, and A. P. Szczepaniak, Phys. Rev. D 93, 034029 (2016)

[71] R. Dashen and R. Rajaraman, Phys. Rev. D 10, 694 (1974).

[72] R. Dashen and R. Rajaraman, Phys. Rev. D 10, 708 (1974).

[73] M. T. Hansen and S. R. Sharpe, Phys. Rev. D 90, 116003 (2014).

[74] R. A. Briceño, M. T. Hansen, and S. R. Sharpe, Phys. Rev. D 95, 074510 (2017).

[75] M. Mai, B. Hu, M. Döring, A. Pilloni, and A. Szczepaniak, Eur. Phys. J. A 53, 177 (2017).

[76] M. Döring, H.-W. Hammer, M. Mai, J.-Y. Pang, A. Rusetsky, and J. Wu, Phys. Rev. D 97, 114508 (2018). 
[77] N. Sharma, J. Cleymans, B. Hippolyte, and M. Paradza, Phys. Rev. C 99, 044914 (2019).

[78] B. Hippolyte and Y. Schutz, https://github.com/thermusproject/THERMUS.

[79] V. Vovchenko, B. Dönigus, and H. Stoecker, Phys. Rev. C 100, 054906 (2019).
[80] U. Loring, B. C. Metsch, and H. R. Petry, Eur. Phys. J. A 10, 447 (2001).

[81] P. Huovinen, P. M. Lo, M. Marczenko, K. Morita, K. Redlich, and C. Sasaki, Phys. Lett. B 769, 509 (2017).

[82] A. Andronic, P. Braun-Munzinger, B. Friman, P. M. Lo, K. Redlich, and J. Stachel, inpreparation (2020). 